human genome. A human proteome would be a very expensive and ambitious undertaking and, by its nature, the full benefits cannot be spelt out beforehand. That's how it should be. The fun of the human genome is that spelling out the letters did little to decipher the code there is so much more complexity than had ever been imagined. We can expect that the proteome will also reveal unexpected delights about the ways in which proteins rally together to perform a task or become tissue.

And yet there was a certain intellectual allure about 'cracking' the human genome that, on the face of it, is lacking from cataloguing all the proteins. And although the human genome is finite, the proteome is almost boundless, because each of the body's proteins may be present in different forms and different amounts in each tissue - and even in precisely the same cell from one moment to the next. That very complexity, however, should inspire, not dissuade.

Proteomic analyses have also been viewed with some scepticism, in part because many studies involving mass-spectrometry profiling have proved difficult to reproduce. And the field has so far largely failed to deliver the disease-tracking biomarkers on which these early efforts were sold. There are few examples of clear, clinically proven benefits. Starting out with this kind of reputation will make a Human Proteome Project particularly hard to sell.

The Human Proteome Organisation (HUPO) has taken some praiseworthy steps towards resolving these issues with, for example, a project to show that different labs can now produce identical results from the same sample. With the rapid evolution of proteomic techniques, the field's reputation and utility is likely to pick up. But HUPO - and the proteomics community - still has a lot to prove and a successful Human Proteome Project is its chance to prove it. It needs to consult widely to devise a strategy that has strong community backing. It will also need to demonstrate that the knowledge stemming from this project will transform the research landscape in the same way that the genome sequence has done.

Failing these, either the project will die or HUPO could risk being left on the sidelines as organizations with money to spend make the decisions about how proteomics should be done.

Times have changed since the Human Genome Project — proposals for mega-biology projects are rather more common and money scarcer. HUPO, and all biologists who love proteins, should articulate clearly and loudly the benefits of sinking US\$1 billion into protein biology. It will take much work to get due recognition for the 'cell's work-horses'.

\section{Superconductors redux}

\section{Yet another surprise has been uncovered in the complex oxides.}

W

ith the discovery of a new class of high-temperature superconductors by reseachers in Japan (see page 922), history seems to be repeating itself. In 1986, Georg Bednorz and Alex Müller of IBM's Zurich research laboratories discovered that a complex oxide of barium, lanthanum and copper became superconducting at $35 \mathrm{~K}$. This sparked an orgy of research that led to the discovery of a related compound (yttrium barium copper oxide) with a superconducting transition temperature of $90 \mathrm{~K}$ : high enough to be attained with relatively cheap liquid-nitrogen cooling. It also won Bednorz and Müller a Nobel prize just a year later.

The excitement stemmed from the prospect of exploiting low-cost superconductivity for loss-free electrical transmission, magnetic levitation and other dazzling applications. In superconductors, currents flow essentially without electrical resistance, the source of energy loss through heating. Before 1986, most superconductors were metals and alloys, with generally paltry transition temperatures that no one had managed to push above $23 \mathrm{~K}$.

Now Hideo Hosono of the Tokyo Institute of Technology and his colleagues have shown that another complex oxide, containing lanthanum, iron, arsenic and a little fluorine, will superconduct at $43 \mathrm{~K}$ when squeezed by around 40,000 atmospheres pressure: a higher temperature than anything bar the copper oxides (see H. Takahashi et al. advance online publication: doi:10.1038/nature06972). Like them, the new material has a sandwich structure of alternate conducting and insulating layers. And like them, doping (replacing some oxygen with fluorine) injects electrons into the conducting

\section{layer that contribute to the supercurrent.}

The copper-oxide materials have found some uses, but nothing to match the expectations heaped on them in the late 1980s - levitating trains and so forth. It has proved hard to fashion these brittle materials into wires and progress is slow. So cynics might grumble that the new breakthrough will merely renew the same unfulfilled promises.

But the new compound already offers more. For one thing, it reveals how much remains to be discovered about complex solid-state compounds. The combinatorial possibilities for four or more elements are so vast that we have barely scratched the surface, despite efforts to automate the search. And as before, the discovery followed from sound chemical intuition. Bednorz and Müller were led to the copper oxides from the apparently unpromising strontium titanium oxide, a superconductor at a mere $0.3 \mathrm{~K}$, by reasoning what kind of crystal chemistry might boost the requisite interactions between electrons. Hosono and his colleagues similarly picked a systematic path from their initial discovery, in 2006, of superconductivity at about $4 \mathrm{~K}$ in a related material, a temperature so low that it attracted little interest. They raised this to $26 \mathrm{~K}$ by the start of 2008 , and rightly figured that squeezing would take it further.

Most crucially, the 'iron oxypnictides' show that high-temperature conductivity is not the sole preserve of copper oxides. As in that case, superconductivity in the new materials seems to be related to magnetic behaviour. But quite how this works has remained a mystery. With an entirely new family of compounds to play with, the mechanism might be persuaded to start giving up some secrets. With a theory to hand, 'designer superconductors' with much higher transition temperatures might not look like a fool's quest. There could be another Nobel prize in that. For now, it is enough that the oxypnictides have set the community buzzing in a way that recalls the last heyday of superconductors two decades ago. 